\title{
EXAMPLES OF DE BRANGES-ROVNYAK SPACES GENERATED BY NONEXTREME FUNCTIONS
}

\author{
Bartosz Łanucha and Maria T. Nowak
}

Maria Curie-Skłodowska University, Institute of Mathematics

pl. M. Curie-Skłodowskiej 1, 20-031 Lublin, Poland; bartosz.lanucha@poczta.umcs.lublin.pl

Maria Curie-Skłodowska University, Institute of Mathematics

pl. M. Curie-Skłodowskiej 1, 20-031 Lublin, Poland; mt.nowak@poczta.umcs.lublin.pl

\begin{abstract}
We describe de Branges-Rovnyak spaces $\mathcal{H}\left(b_{\alpha}\right), \alpha>0$, where the function $b_{\alpha}$ is not extreme in the unit ball of $H^{\infty}$ on the unit disk $\mathbf{D}$, defined by the equality $b_{\alpha}(z) / a_{\alpha}(z)=(1-z)^{-\alpha}$, $z \in \mathbf{D}$, where $a_{\alpha}$ is the outer function such that $a_{\alpha}(0)>0$ and $\left|a_{\alpha}\right|^{2}+\left|b_{\alpha}\right|^{2}=1$ a.e. on $\partial \mathbf{D}$.
\end{abstract}

\section{Introduction}

Let $H^{2}$ denote the standard Hardy space in the open unit disk $\mathbf{D}$ and let $\mathbf{T}=$ $\partial \mathbf{D}$. For $\chi \in L^{\infty}(\mathbf{T})$ let $T_{\chi}$ denote the bounded Toeplitz operator on $H^{2}$, that is, $T_{\chi} f=P_{+}(\chi f)$, where $P_{+}$is the orthogonal projection of $L^{2}(\mathbf{T})$ onto $H^{2}$. In particular, $S=T_{z}$ is called the shift operator. We will denote by $\mathcal{M}(\chi)$ the range of $T_{\chi}$ equipped with the range norm, that is, the norm that makes the operator $T_{\chi}$ a coisometry of $H^{2}$ onto $\mathcal{M}(\chi)$.

Let $\mathcal{S}$ denote the closed unit ball of $H^{\infty}$, that is, $\mathcal{S}=\left\{f \in H^{\infty}:\|f\|_{\infty} \leq 1\right\}$. Let us recall that $f \in \mathcal{S}$ is an extreme point of $\mathcal{S}$ if it is not a proper convex combination of two different elements of $\mathcal{S}$. It is known that $f \in \mathcal{S}$ is an extreme point of $\mathcal{S}$ if and only if

$$
\int_{0}^{2 \pi} \log \left(1-\left|f\left(e^{i t}\right)\right|\right) d t=-\infty
$$

(see [3, pp. 125-127] and [6, Thm. 6.7]).

Given a function $b$ in $\mathcal{S}$, the de Branges-Rovnyak space $\mathcal{H}(b)$ is the image of $H^{2}$ under the operator $\left(I-T_{b} T_{\bar{b}}\right)^{1 / 2}$ with the corresponding range norm $\|\cdot\|_{b}$. The space $\mathcal{H}(b)$ is a Hilbert space with reproducing kernel

$$
k_{w}^{b}(z)=\frac{1-\overline{b(w)} b(z)}{1-\bar{w} z} \quad(z, w \in \mathbf{D}) .
$$

Here we are interested in the case when the function $b$ is not an extreme point of $\mathcal{S}$, that is, when $\log (1-|b|) \in L^{1}(\mathbf{T})$. Then there exists an outer function $a \in H^{\infty}$ for which $|a|^{2}+|b|^{2}=1$ a.e. on $\mathbf{T}$. Moreover, if we suppose that $a(0)>0$, then $a$ is uniquely determined, and, following Sarason, we say that $(b, a)$ is a pair. The function $a$ is sometimes called the Pythagorean mate associated with $b$ (see [6, Vol. 2, p. 274]).

It is known that both $\mathcal{M}(a)$ and $\mathcal{M}(\bar{a})$ are contained contractively in $\mathcal{H}(b)$ (see $[12$, p. 25]). Moreover, if $(b, a)$ is a corona pair, that is, $|a|+|b|$ is bounded away from 0 in $\mathbf{D}$, then $\mathcal{H}(b)=\mathcal{M}(\bar{a})$ (see e.g. [12, p. 62]).

https://doi.org/10.5186/aasfm.2019.4427

2010 Mathematics Subject Classification: Primary 47B32, 30H10, 30H15.

Key words: Hardy space, de Branges-Rovnyak space, Smirnov class, rigid function. 
Let us recall that the Smirnov class $\mathcal{N}^{+}$consists of those holomorphic functions in $\mathbf{D}$ that are quotients of functions in $H^{\infty}$ in which the denominators are outer functions. If $(b, a)$ is a pair, then the quotient $\varphi=b / a$ is in $\mathcal{N}^{+}$, and conversely, for every nonzero function $\varphi \in \mathcal{N}^{+}$there exists a unique pair $(b, a)$ such that $\varphi=b / a$ ([14]).

Many properties of $\mathcal{H}(b)$ can be expressed in terms of the function $\varphi=b / a$ in the Smirnov class $\mathcal{N}^{+}$. It is worth noting here that if $\varphi$ is rational, then the functions $a$ and $b$ in the representation of $\varphi$ are also rational (see [14]) and in such a case $(b, a)$ is called a rational pair. Spaces $\mathcal{H}(b)$ for rational pairs have been studied in [13], [1], [2], [4] and [8] where, among other results, the connection between $\mathcal{H}(b)$ and the local Dirichlet spaces has been discussed. Recently, in [4], the authors studied also the spaces $\mathcal{H}\left(b^{r}\right)$, where $b$ is a rational outer function in the closed unit ball of $H^{\infty}$ and $r$ is a positive number.

Here we describe the Branges-Rovnyak spaces $\mathcal{H}\left(b_{\alpha}\right), \alpha>0$, where $\left(b_{\alpha}, a_{\alpha}\right)$ is such a pair that

(principal branch).

$$
\varphi_{\alpha}(z)=\frac{b_{\alpha}(z)}{a_{\alpha}(z)}=\frac{1}{(1-z)^{\alpha}}
$$

Following Sarason [14], for a function $\varphi$ that is holomorphic on $\mathbf{D}$ we define $T_{\varphi}$ to be the operator of multiplication by $\varphi$ on the domain $\mathcal{D}\left(T_{\varphi}\right)=\left\{f \in H^{2}: \varphi f \in H^{2}\right\}$. It is easy to verify that $T_{\varphi}$ is a closed operator (see [6, Vol. 2, p. 309]). It was proved in [14] that the domain $\mathcal{D}\left(T_{\varphi}\right)$ is dense in $H^{2}$ if and only if $\varphi \in \mathcal{N}^{+}$. More precisely, if $\varphi$ is a nonzero function in $\mathcal{N}^{+}$with canonical representation $\varphi=b / a$, then $\mathcal{D}\left(T_{\varphi}\right)=a H^{2}$. In this case $T_{\varphi}$ has a unique, densely defined adjoint $T_{\varphi}^{*}$ that is also closed. In what follows we denote $T_{\bar{\varphi}}=T_{\varphi}^{*}$. The reason for such a notation for $T_{\varphi}^{*}$ is explained in [14, pp. 286-288]. The next theorem says that the domain of $T_{\bar{\varphi}}$ coincides with the de Branges-Rovnyak space $\mathcal{H}(b)$.

Theorem 1.1. [14], [6, Thm. 23.31] Let $(b, a)$ be a pair and let $\varphi=b / a$. Then the domain of $T_{\bar{\varphi}}$ is $\mathcal{H}(b)$ and for $f \in \mathcal{H}(b)$,

$$
\|f\|_{b}^{2}=\|f\|_{2}^{2}+\left\|T_{\bar{\varphi}} f\right\|_{2}^{2} .
$$

The next proposition was also proved in [14].

Proposition 1.2. [14] If $\varphi$ is in $\mathcal{N}^{+}, \psi$ is in $H^{\infty}$, and $f$ is in $\mathcal{D}\left(T_{\bar{\varphi}}\right)$, then

$$
T_{\bar{\varphi}} T_{\bar{\psi}} f=T_{\overline{\varphi \psi}} f=T_{\bar{\psi}} T_{\bar{\varphi}} f
$$

Corollary 1.3. Let $\varphi_{1}, \varphi_{2} \in \mathcal{N}^{+}$have canonical representations $\varphi_{i}=b_{i} / a_{i}$, $i=1$, 2. If $\varphi_{2} / \varphi_{1} \in H^{\infty}$, then $\mathcal{H}\left(b_{1}\right) \subset \mathcal{H}\left(b_{2}\right)$. and so

Proof. Put $\psi=\varphi_{2} / \varphi_{1}$. It follows from Proposition 1.2 that $\mathcal{D}\left(T_{\bar{\varphi}_{1}}\right) \subset \mathcal{D}\left(T_{\overline{\varphi_{1} \psi}}\right)$,

$$
\mathcal{H}\left(b_{1}\right)=\mathcal{D}\left(T_{\bar{\varphi}_{1}}\right) \subset \mathcal{D}\left(T_{\overline{\varphi_{1} \psi}}\right)=\mathcal{D}\left(T_{\bar{\varphi}_{2}}\right)=\mathcal{H}\left(b_{2}\right) .
$$

In the proof of our main theorem we will use the following description of invertible Toeplitz operators with unimodular symbols.

Devinatz-Widom Theorem. [9, p. 250] Let $\psi \in L^{\infty}(\partial \mathbf{D})$ be such that $|\psi|=1$ a.e. on $\partial \mathbf{D}$. The following are equivalent.

(a) $T_{\psi}$ is invertible.

(b) $\operatorname{dist}\left(\psi, H^{\infty}\right)<1$ and $\operatorname{dist}\left(\bar{\psi}, H^{\infty}\right)<1$.

(c) There exists an outer function $h \in H^{\infty}$ such that $\|\psi-h\|_{\infty}<1$. 
(d) There exist real valued bounded functions $u, v$ and a constant $c \in \mathbf{R}$ such that $\psi=e^{i(u+\tilde{v}+c)}$ and $\|u\|_{\infty}<\frac{\pi}{2}$, where $\tilde{v}$ denotes the conjugate function of $v$.

We will need also the notion of a rigid function in $H^{1}$. A function in $H^{1}$ is called rigid if no other functions in $H^{1}$, except for positive scalar multiples of itself, have the same argument as it almost everywhere on $\partial \mathbf{D}$. As observed in [11], every rigid function is outer. It is known that the function $(1-z)^{\alpha}$ is rigid if $0<\alpha \leq 1$ and is not rigid if $\alpha>1$ (see e.g. [6, Section 6.8]).

The next theorem shows a close connection between kernels of Toeplitz operators and rigid functions in $H^{1}$ ([12, p. 70]).

Theorem 1.4. If $f$ is an outer function in $H^{2}$, then $f^{2}$ is rigid if and only if the operator $T_{\bar{f} / f}$ has a trivial kernel.

Moreover, for a pair $(b, a)$ the following sufficient condition for density of $\mathcal{M}(a)$ in $\mathcal{H}(b)$ is known ([12, p. 72], [6, vol. 2, p. 496]).

Theorem 1.5. If the function $a^{2}$ is rigid, then $\mathcal{M}(a)$ is dense in $\mathcal{H}(b)$.

\section{The spaces $\mathcal{H}\left(b_{\alpha}\right), \alpha>0$}

Recall that for $\alpha>0$ we define the pair $\left(b_{\alpha}, a_{\alpha}\right)$ by

$$
\varphi_{\alpha}(z)=\frac{b_{\alpha}(z)}{a_{\alpha}(z)}=\frac{1}{(1-z)^{\alpha}} .
$$

Consequently, the outer function $a_{\alpha}$ is given by

$$
a_{\alpha}(z)=\exp \left\{\frac{1}{4 \pi} \int_{0}^{2 \pi} \frac{e^{i t}+z}{e^{i t}-z} \log \frac{\left|1-e^{i t}\right|^{2 \alpha}}{1+\left|1-e^{i t}\right|^{2 \alpha}} d t\right\} .
$$

Since both $a_{\alpha}$ and $(1-z)^{\alpha}$ are outer functions, by the uniqueness of inner-outer factorization, the equality $(1-z)^{\alpha} b_{\alpha}(z)=a_{\alpha}(z)$ implies that $b_{\alpha}$ is also outer. Hence

$$
b_{\alpha}(z)=a_{\alpha}(z) \varphi_{\alpha}(z)=\exp \left\{\frac{1}{4 \pi} \int_{0}^{2 \pi} \frac{e^{i t}+z}{e^{i t}-z} \log \frac{1}{1+\left|1-e^{i t}\right|^{2 \alpha}} d t\right\} .
$$

This formula shows that $\log \left|b_{\alpha}(z)\right|$ is a function harmonic in $\mathbf{D}$ and continuous in $\overline{\mathbf{D}}$ with $\log \left|b_{\alpha}\left(e^{i t}\right)\right|^{2}=\log \frac{1}{1+\left|1-e^{i t}\right|^{2 \alpha}}$. In particular, $\log \left|b_{\alpha}(1)\right|=\log 1=0$. We now prove that actually $b_{\alpha}(1)=1$. To this end, it is enough to note that $\arg b_{\alpha}(r)=0$ for all $0<r<1$. Indeed,

$$
\begin{aligned}
\arg b_{\alpha}(r) & =\frac{1}{4 \pi} \int_{0}^{2 \pi} \operatorname{Im}\left(\frac{e^{i t}+r}{e^{i t}-r}\right) \log \frac{1}{1+\left|1-e^{i t}\right|^{2 \alpha}} d t \\
& =-\frac{1}{4 \pi} \int_{-\pi}^{\pi} \frac{2 r \sin t}{\left|e^{i t}-r\right|^{2}} \log \frac{1}{1+\left|1-e^{i t}\right|^{2 \alpha}} d t=0
\end{aligned}
$$

because the integrand is an odd function.

The following proposition says for which $\alpha$ a nontangential limit at 1 of each function (and its derivatives up to a given order) from $\mathcal{H}\left(b_{\alpha}\right)$ exists.

Proposition 2.1. Every $f \in \mathcal{H}\left(b_{\alpha}\right)$ along with its derivatives up to order $n-1$ has a nontangential limit at the point 1 if and only if $n<\alpha+1 / 2$.

This is a consequence of Theorem 3.2 from [7] (see also [12] and [4]), which states that the following two conditions are equivalent: 
(i) for every $f \in \mathcal{H}\left(b_{\alpha}\right)$ the functions $f(z), f^{\prime}(z), \ldots, f^{(n-1)}(z)$ have finite limits as $z$ tends nontangentially to 1 ;

(ii)

$$
\int_{0}^{2 \pi} \frac{|\log | b_{\alpha}\left(e^{i t}\right)||}{\left|1-e^{i t}\right|^{2 n}} d t<+\infty
$$

Since

$$
\log \left|b_{\alpha}\left(e^{i t}\right)\right|^{2}=\log \frac{1}{1+\left|1-e^{i t}\right|^{2 \alpha}}=\log \left(1-\frac{\left|1-e^{i t}\right|^{2 \alpha}}{1+\left|1-e^{i t}\right|^{2 \alpha}}\right)
$$

and $|\log (1-x)| \approx|x|$ for $x$ sufficiently close to zero, we have

$$
\log \left|b_{\alpha}\left(e^{i t}\right)\right| \approx \frac{\left|1-e^{i t}\right|^{2 \alpha}}{1+\left|1-e^{i t}\right|^{2 \alpha}} \approx\left|1-e^{i t}\right|^{2 \alpha}
$$

whenever $t$ is sufficiently close to 0 or $2 \pi$. This implies that

$$
\int_{0}^{2 \pi} \frac{|\log | b_{\alpha}\left(e^{i t}\right)||}{\left|1-e^{i t}\right|^{2 n}} d t<\infty
$$

if and only if

$$
\int_{0}^{2 \pi} \frac{1}{\left|1-e^{i t}\right|^{2 n-2 \alpha}} d t<\infty
$$

which holds exactly when $\alpha>n-1 / 2$.

In particular, we see that every $f \in \mathcal{H}\left(b_{\alpha}\right)$ has a nontangential limit at 1 if and only if $\alpha>1 / 2$.

The next proposition is an immediate consequence of Corollary 1.3.

Proposition 2.2. For every $0<\alpha \leq \beta<\infty$,

$$
\mathcal{H}\left(b_{\beta}\right) \subset \mathcal{H}\left(b_{\alpha}\right) .
$$

Observe now that $b_{\alpha}$ is bounded below. Indeed, by (2.2),

$$
\begin{aligned}
\left|b_{\alpha}(z)\right| & =\exp \left\{\frac{1}{4 \pi} \int_{0}^{2 \pi} \operatorname{Re} \frac{e^{i t}+z}{e^{i t}-z} \log \frac{1}{1+\left|1-e^{i t}\right|^{2 \alpha}} d t\right\} \\
& \geq \exp \left\{\log \sqrt{\frac{1}{1+4^{\alpha}}} \cdot \frac{1}{2 \pi} \int_{0}^{2 \pi} \operatorname{Re} \frac{e^{i t}+z}{e^{i t}-z} d t\right\}=\sqrt{\frac{1}{1+4^{\alpha}}} .
\end{aligned}
$$

Clearly, this implies that $\left(b_{\alpha}, a_{\alpha}\right)$ is a corona pair for $\alpha>0$.

Corollary 2.3. For $\alpha>0$,

$$
\mathcal{M}\left(a_{\alpha}\right)=\mathcal{M}\left((1-z)^{\alpha}\right) \quad \text { and } \quad \mathcal{H}\left(b_{\alpha}\right)=\mathcal{M}\left(\bar{a}_{\alpha}\right)=\mathcal{M}\left(\overline{(1-z)^{\alpha}}\right)
$$

with equivalence of norms.

Proof. The equality of $\mathcal{H}\left(b_{\alpha}\right)$ and $\mathcal{M}\left(\bar{a}_{\alpha}\right)$ follows from the fact that $\left(b_{\alpha}, a_{\alpha}\right)$ is a corona pair, which as noted above is a consequence of the fact that $b_{\alpha}$ is bounded below. The latter implies that $1 / b_{\alpha} \in H^{\infty}$ and so $T_{b_{\alpha}}$ and $T_{\bar{b}_{\alpha}}$ are invertible. Hence

$$
\mathcal{M}\left((1-z)^{\alpha}\right)=T_{\frac{a_{\alpha}}{b_{\alpha}}} H^{2}=T_{a_{\alpha}} H^{2}
$$

and

$$
\mathcal{M}\left(\overline{(1-z)^{\alpha}}\right)=T_{\frac{\bar{a}_{\alpha}}{\bar{b}_{\alpha}}} H^{2}=T_{\bar{a}_{\alpha}} H^{2} .
$$

Both $\mathcal{M}\left(a_{\alpha}\right)$ and $\mathcal{M}\left((1-z)^{\alpha}\right)$ are boundedly contained in $H^{2}$. Hence, the Closed Graph Theorem implies equivalence of their norms. Similarly, one obtains the equivalence of norms in $\mathcal{M}\left(\bar{a}_{\alpha}\right)$ and $\mathcal{M}\left(\overline{(1-z)^{\alpha}}\right)$. 
It is worth mentioning here that many results on the space $\mathcal{M}(\bar{a})$ where $a \in H^{\infty}$ is an outer function have been recently obtained in [5]. In this paper the authors study, in particular, boundary behavior of the functions from $\mathcal{M}(\bar{a})$ and describe a natural orthogonal decomposition of this space.

\section{Main results}

We start with the following.

Theorem 3.1. For any $n \in \mathbf{N}$ and $n-1 / 2<\alpha<n+1 / 2$ we have

$$
\mathcal{M}\left(\overline{(1-z)^{\alpha}}\right)=\mathcal{M}\left((1-z)^{\alpha}\right)+\operatorname{span}\left\{S^{*}(1-z)^{\alpha}, \ldots, S^{* n}(1-z)^{\alpha}\right\} .
$$

Proof. Let

$$
Q(z)=\frac{1-z}{1-\bar{z}}, \quad z \in \mathbf{D} .
$$

Then $Q$ has a continuous extension to $\overline{\mathbf{D}} \backslash\{1\}$ and

$$
Q(z)=-z \quad \text { for } z \in \mathbf{T} \backslash\{1\}
$$

which implies that

$$
T_{Q^{n}}=(-1)^{n} S^{n} \quad \text { for } n \geq 1 .
$$

Moreover, we observe that for $n-1 / 2<\alpha<n+1 / 2, n \geq 1$, we have

$$
T_{Q^{\alpha}}=T_{Q^{\alpha-n} Q^{n}}=(-1)^{n} T_{Q^{\alpha-n}} S^{n} .
$$

Consequently,

$$
T_{(1-z)^{\alpha}}=T_{\overline{(1-z)^{\alpha}} Q^{\alpha}}=(-1)^{n} T_{\overline{(1-z)^{\alpha}}} T_{Q^{\alpha-n}} S^{n} .
$$

Observe now that the operator $T_{Q^{\alpha-n}}$ is invertible. Indeed, we have

$$
Q^{\alpha-n}\left(e^{i t}\right)=e^{i(\alpha-n)(t-\pi)}, \quad t \in(0,2 \pi),
$$

where $|\alpha-n|<1 / 2$. So invertibility of $T_{Q^{\alpha-n}}$ follows from part (d) of the DevinatzWidom Theorem.

Let $f \in \mathcal{M}\left(\overline{(1-z)^{\alpha}}\right)$ and $f=T_{\overline{(1-z)^{\alpha}}} g$ for a function $g \in H^{2}$. Since $T_{Q^{\alpha-n}}$ is invertible, there exists $g_{0} \in H^{2}$ such that $(-1)^{n} g=T_{Q^{\alpha-n}} g_{0}$. Hence, using (3.2), we obtain

$$
\begin{aligned}
f=T_{\overline{(1-z)^{\alpha}}} g & =(-1)^{n} T_{\overline{(1-z)^{\alpha}}} T_{Q^{\alpha-n}} g_{0} \\
& =(-1)^{n} T_{\overline{(1-z)^{\alpha}}} T_{Q^{\alpha-n}}\left(S^{n} S^{* n} g_{0}+\sum_{k=0}^{n-1}\left\langle g_{0}, z^{k}\right\rangle z^{k}\right) \\
& =T_{(1-z)^{\alpha}} S^{* n} g_{0}+(-1)^{n} \sum_{k=0}^{n-1}\left\langle g_{0}, z^{k}\right\rangle T_{\overline{(1-z)^{\alpha}}} T_{Q^{\alpha-n}} z^{k} .
\end{aligned}
$$

Since for $0 \leq k \leq n-1$,

$$
\begin{aligned}
(-1)^{n} T_{\overline{(1-z)^{\alpha}}} T_{Q^{\alpha-n}} z^{k} & =(-1)^{n} T_{\bar{Q}^{n}(1-z)^{\alpha}} S^{k} 1 \\
& =S^{*(n-k)} T_{(1-z)^{\alpha}} 1=S^{*(n-k)}(1-z)^{\alpha} \quad \text { by }(3.1),
\end{aligned}
$$

we get

$$
\begin{aligned}
f= & (1-z)^{\alpha} S^{* n} g_{0}+\sum_{k=0}^{n-1}\left\langle g_{0}, z^{k}\right\rangle S^{*(n-k)}(1-z)^{\alpha} \\
& \in \mathcal{M}\left((1-z)^{\alpha}\right)+\operatorname{span}\left\{S^{*}(1-z)^{\alpha}, \ldots, S^{* n}(1-z)^{\alpha}\right\} .
\end{aligned}
$$


To show that

$$
\mathcal{M}\left((1-z)^{\alpha}\right)+\operatorname{span}\left\{S^{*}(1-z)^{\alpha}, \ldots, S^{* n}(1-z)^{\alpha}\right\} \subset \mathcal{M}\left(\overline{(1-z)^{\alpha}}\right),
$$

it is enough to observe that $\mathcal{M}\left((1-z)^{\alpha}\right) \subset \mathcal{M}\left(\overline{(1-z)^{\alpha}}\right)$ and $\mathcal{M}\left(\overline{(1-z)^{\alpha}}\right)$ is $S^{*}$ invariant.

Now we prove our main result.

Theorem 3.2. Let $0<\alpha<\infty$ and let $\left(b_{\alpha}, a_{\alpha}\right)$ be a pair, with the functions $b_{\alpha}$ and $a_{\alpha}$ given by (2.2) and (2.1), respectively. Then

(i) for $0<\alpha<1 / 2$,

$$
\mathcal{H}\left(b_{\alpha}\right)=\mathcal{M}\left(a_{\alpha}\right)=(1-z)^{\alpha} H^{2},
$$

(ii) for $n-1 / 2<\alpha<n+1 / 2, n=1,2, \ldots$,

$$
\mathcal{H}\left(b_{\alpha}\right)=\mathcal{M}\left(a_{\alpha}\right)+\mathcal{P}_{n}=(1-z)^{\alpha} H^{2}+\mathcal{P}_{n},
$$

where $\mathcal{P}_{n}$ is the set of all polynomials of degree at most $n-1$,

(iii)

$$
\mathcal{H}\left(b_{1 / 2}\right)=\overline{\mathcal{M}\left(a_{1 / 2}\right)}=\overline{(1-z)^{1 / 2} H^{2}},
$$

where the closure is taken with respect to the $\mathcal{H}\left(b_{1 / 2}\right)$-norm,

(iv) for $\alpha=n+1 / 2, n=1,2, \ldots$,

$$
\mathcal{H}\left(b_{\alpha}\right)=\overline{\mathcal{M}\left(a_{\alpha}\right)}+\mathcal{A}_{n},
$$

where the closure is taken with respect to the $\mathcal{H}\left(b_{\alpha}\right)$-norm and $\mathcal{A}_{n}$ is the $n$-dimensional subspace of $\mathcal{H}\left(b_{\alpha}\right)$ defined by

$$
\mathcal{A}_{n}=\left\{p_{n} \cdot P_{+}\left(\overline{(1-z)^{\alpha}}(1-z)^{1 / 2}\right)+P_{+}\left(p_{n} P_{-}\left(\overline{(1-z)^{\alpha}}(1-z)^{1 / 2}\right)\right): p_{n} \in \mathcal{P}_{n}\right\}
$$

where $P_{-}=I-P_{+}$.

Proof. (i) We know from Corollary 2.3 that for $\alpha>0$,

$$
\mathcal{H}\left(b_{\alpha}\right)=\mathcal{M}\left(\bar{a}_{\alpha}\right)=\mathcal{M}\left(\overline{(1-z)^{\alpha}}\right) .
$$

As in the proof of Theorem 3.1 it follows from the Devinatz-Widom Theorem that for $0<\alpha<1 / 2$ the operator $T_{Q^{\alpha}}$ is invertible. Consequently,

$$
\mathcal{M}\left(\overline{(1-z)^{\alpha}}\right)=T_{\overline{(1-z)^{\alpha}}} H^{2}=T_{\overline{(1-z)^{\alpha}}} T_{Q^{\alpha}} H^{2}=(1-z)^{\alpha} H^{2} .
$$

(ii) Since $\mathcal{H}\left(b_{\alpha}\right)$ contains $\mathcal{M}\left(a_{\alpha}\right)=\mathcal{M}\left((1-z)^{\alpha}\right)$ and all polynomials (see e.g. [12, p. 25]), to prove (ii) it is enough to show that

$$
\mathcal{H}\left(b_{\alpha}\right) \subset \mathcal{P}_{n}+\mathcal{M}\left((1-z)^{\alpha}\right) .
$$

By Theorem 3.1 we have

$$
\mathcal{H}\left(b_{\alpha}\right)=\mathcal{M}\left(\overline{(1-z)^{\alpha}}\right)=\mathcal{M}\left((1-z)^{\alpha}\right)+\operatorname{span}\left\{S^{*}(1-z)^{\alpha}, \ldots, S^{* n}(1-z)^{\alpha}\right\} .
$$

Therefore, we only need to show that

$$
\operatorname{span}\left\{S^{*}(1-z)^{\alpha}, \ldots, S^{* n}(1-z)^{\alpha}\right\} \subset \mathcal{P}_{n}+\mathcal{M}\left((1-z)^{\alpha}\right) .
$$

Clearly,

$$
\begin{aligned}
S^{*}(1-z)^{\alpha} & =\frac{(1-z)^{\alpha}-1}{z}=\frac{(1-z)^{\alpha}-(1-z)^{n}+(1-z)^{n}-1}{z} \\
& =S^{*}(1-z)^{n}-(1-z)^{\alpha} S^{*}(1-z)^{n-\alpha} \in \mathcal{P}_{n}+\mathcal{M}\left((1-z)^{\alpha}\right)
\end{aligned}
$$


$\left((1-z)^{n-\alpha} \in H^{2}\right.$ since $\left.n-\alpha>-1 / 2\right)$. Now assume that for any $1 \leq k<n$,

$$
S^{* k}(1-z)^{\alpha} \in \mathcal{P}_{n}+\mathcal{M}\left((1-z)^{\alpha}\right),
$$

or, in other words,

$$
S^{* k}(1-z)^{\alpha}=p_{n}+(1-z)^{\alpha} h_{k} \text { for some } p_{n} \in \mathcal{P}_{n} \text { and } h_{k} \in H^{2} .
$$

Then

$$
\begin{aligned}
S^{*(k+1)}(1-z)^{\alpha} & =S^{*}\left(S^{* k}(1-z)^{\alpha}\right)=\frac{p_{n}+(1-z)^{\alpha} h_{k}-p_{n}(0)-h_{k}(0)}{z} \\
& =\frac{p_{n}+(1-z)^{\alpha} h_{k}-(1-z)^{\alpha} h_{k}(0)+(1-z)^{\alpha} h_{k}(0)-p_{n}(0)-h_{k}(0)}{z} \\
& =S^{*} p_{n}+h_{k}(0) S^{*}(1-z)^{\alpha}+(1-z)^{\alpha} S^{*} h_{k} \in \mathcal{P}_{n}+\mathcal{M}\left((1-z)^{\alpha}\right) .
\end{aligned}
$$

This completes the proof of (ii).

(iii) In view of Theorem 1.5, to prove (iii) it is enough to show that $a_{1 / 2}^{2}$ is a rigid function. We actually prove that $a_{\alpha}^{2}$ is rigid for every $0<\alpha \leq 1 / 2$. To this end, we observe that by (2.3), for $\alpha>0$,

$$
\frac{1}{\sqrt{1+4^{\alpha}}}|1-z|^{\alpha} \leq\left|a_{\alpha}(z)\right| \leq|1-z|^{\alpha}, \quad z \in \mathbf{D} .
$$

and so $(1-z)^{\alpha} / a_{\alpha} \in H^{\infty}$.

Now we use a reasoning analogous to that in $[12,(\mathrm{X}-5)]$. If $a_{\alpha}^{2}$ is not rigid for some $0<\alpha \leq 1 / 2$, then by Theorem 1.4 there is a nonzero function $g$ in the kernel of $T_{\bar{a}_{\alpha} / a_{\alpha}}$. Then

$$
T_{\frac{\overline{(1-z)^{\alpha}}}{(1-z)^{\alpha}}}\left(\frac{(1-z)^{\alpha} g}{a_{\alpha}}\right)=P_{+}\left(\frac{\overline{(1-z)^{\alpha}} g}{a_{\alpha}}\right)=P_{+}\left(\frac{\overline{(1-z)^{\alpha}} g}{\overline{a_{\alpha}}} \cdot \frac{\bar{a}_{\alpha}}{a_{\alpha}}\right)=T_{\frac{\overline{(1-z)^{\alpha}}}{\bar{a}_{\alpha}}} T_{\frac{\bar{a}_{\alpha}}{a_{\alpha}}} g=0,
$$

which means that $(1-z)^{\alpha} g / a_{\alpha} \in H^{2}$ is a nonzero function in the kernel of $T \overline{(1-z)^{\alpha}} /(1-z)^{\alpha}$, contrary to the fact that $(1-z)^{2 \alpha}$ is rigid for $0<\alpha \leq 1 / 2$ (see, e.g., [6, Section 6.8]).

(iv) We know that for every $\alpha>0$,

$$
\mathcal{H}\left(b_{\alpha}\right)=\mathcal{M}\left(\bar{a}_{\alpha}\right)=\mathcal{M}\left(\overline{(1-z)^{\alpha}}\right)=T \overline{(1-z)^{\alpha}} H^{2}
$$

and $\mathcal{M}\left(a_{\alpha}\right)=\mathcal{M}\left((1-z)^{\alpha}\right)$ is the image under $T_{\overline{(1-z)^{\alpha}}}$ of the range of $T_{(1-z)^{\alpha} / \overline{(1-z)^{\alpha}}}$, that is,

$$
\mathcal{M}\left((1-z)^{\alpha}\right)=T_{\overline{(1-z)^{\alpha}}} T_{\frac{(1-z)^{\alpha}}{(1-z)^{\alpha}}} H^{2} .
$$

It follows that the orthogonal complement of $\mathcal{M}\left((1-z)^{\alpha}\right)$ in the space $\mathcal{M}\left(\overline{(1-z)^{\alpha}}\right)$ is the image under $T \overline{(1-z)^{\alpha}}$ of $\operatorname{ker} T \overline{(1-z)^{\alpha}} /(1-z)^{\alpha}$.

Clearly, for $\alpha=n+1 / 2$ we have

$$
\operatorname{ker} T_{\frac{(1-z)^{\alpha}}{(1-z)^{\alpha}}}=\operatorname{ker} T_{z^{n}} T_{\frac{(1-z)^{1 / 2}}{(1-z)^{1 / 2}}} .
$$

Since $1-z$ is a rigid function, we get

$$
\operatorname{ker} T_{\overline{z^{n}}} T \frac{}{\frac{(1-z)^{1 / 2}}{(1-z)^{1 / 2}}}=(1-z)^{1 / 2} \mathcal{P}_{n}
$$

where $\mathcal{P}_{n}$ is the set of all polynomials of degree at most $n-1$. Finally, note that if $p_{n}$ is in $\mathcal{P}_{n}$, then

$$
\begin{aligned}
& T_{\overline{(1-z)^{\alpha}}}\left((1-z)^{1 / 2} p_{n}\right)=P_{+}\left(\overline{(1-z)^{\alpha}}(1-z)^{1 / 2} p_{n}\right) \\
& =P_{+}\left(\overline{(1-z)^{\alpha}}(1-z)^{1 / 2}\right) p_{n}+P_{+}\left(P_{-}\left(\overline{(1-z)^{\alpha}}(1-z)^{1 / 2}\right) p_{n}\right) .
\end{aligned}
$$


Our claim follows.

The following corollary is just another statement of (ii) in Theorem 3.2.

Corollary 3.3. For any $n \in \mathbf{N}$ and $n-1 / 2<\alpha<n+1 / 2$ we have

$$
\mathcal{H}\left(b_{\alpha}\right)=\mathcal{M}\left(a_{\alpha}\right)+\mathcal{P}_{n}=\mathcal{M}\left(a_{\alpha}\right)+\operatorname{span}\left\{T_{\bar{a}_{\alpha}} 1, \ldots, T_{\bar{a}_{\alpha}} z^{n-1}\right\} .
$$

Remark 3.4. We observe that since $a_{\alpha}^{2}$ is rigid for all $0<\alpha \leq 1 / 2$, Theorem 1.5 implies that the space $\mathcal{M}\left(a_{\alpha}\right)$ is dense in $\mathcal{H}\left(b_{\alpha}\right)$ for all such $\alpha$. However, for $0<$ $\alpha<1 / 2$ we have $\mathcal{M}\left(a_{\alpha}\right)=\mathcal{H}\left(b_{\alpha}\right)$, while $\mathcal{M}\left(a_{1 / 2}\right) \subsetneq \mathcal{H}\left(b_{1 / 2}\right)$. The latter follows from the fact that every $h \in H^{2}$ satisfies $|h(z)|=o\left((1-|z|)^{-1 / 2}\right)$ as $|z| \rightarrow 1^{-}$. Thus if $f \in \mathcal{M}\left(a_{1 / 2}\right)$, then $f(z)=(1-z)^{1 / 2} h(z), h \in H^{2}$, and

$$
|f(z)|=|1-z|^{\frac{1}{2}}|h(z)|=\left(\frac{|1-z|}{1-|z|}\right)^{\frac{1}{2}}|h(z)|(1-|z|)^{\frac{1}{2}} .
$$

This shows that the nontangential limit of $f$ at 1 is 0 . On the other hand, $\mathcal{H}\left(b_{1 / 2}\right)$ contains nonzero constants, so $\mathcal{M}\left(a_{1 / 2}\right)$ cannot be equal to $\mathcal{H}\left(b_{1 / 2}\right)$.

Corollary 3.5. If $n-1 / 2<\alpha<n+1 / 2, n \in \mathbf{N}$, and $f \in \mathcal{H}\left(b_{\alpha}\right)$, then there is a function $h$ in $H^{2}$ such that

$$
f(z)=f(1)+f^{\prime}(1)(z-1)+\ldots+\frac{f^{(n-1)}(1)}{(n-1) !}(z-1)^{n-1}+(1-z)^{\alpha} h(z) .
$$

Proof. It follows from Proposition 2.1 that $f$ and its derivatives of order up to $n-1$ have nontangential limits at 1 , say $f(1), f^{\prime}(1), \ldots, f^{(n-1)}(1)$. By Theorem 3.2(ii), $f$ can be written as

$$
f(z)=p_{n}(z)+(1-z)^{\alpha} h(z)=\sum_{k=0}^{n-1} a_{k}(z-1)^{k}+(1-z)^{\alpha} h(z), \quad h \in H^{2} .
$$

Since every $h$ in $H^{2}$ satisfies

$$
\left|h^{(k)}(z)\right| \leq \frac{c_{k}}{(1-|z|)^{k+\frac{1}{2}}}
$$

we find that

$$
a_{k}=\frac{p_{n}^{(k)}(1)}{k !}=\frac{f^{(k)}(1)}{k !} \text { for } k=0,1, \ldots, n-1 .
$$

The next theorem describes the space $\mathcal{H}\left(\tilde{b}_{\alpha}\right)$ where $\tilde{b}_{\alpha}$ is an outer function from the unit ball of $H^{\infty}$ whose Pythagorean mate is $\left(\frac{1-z}{2}\right)^{\alpha}, \alpha>0$.

Theorem 3.6. For $\alpha>0$ let $\tilde{a}_{\alpha}(z)=\left(\frac{1-z}{2}\right)^{\alpha}$ and let $\tilde{b}_{\alpha}$ be the outer function such that $\left(\tilde{b}_{\alpha}, \tilde{a}_{\alpha}\right)$ is a pair. Then

$$
\mathcal{H}\left(\tilde{b}_{\alpha}\right)=\mathcal{H}\left(b_{\alpha}\right) .
$$

Proof. It is enough to show that $\left(\tilde{b}_{\alpha}, \tilde{a}_{\alpha}\right)$ is a corona pair. We will use the reasoning similar to that in the proof of Lemma 3.3 in [4]. The function $\tilde{a}_{\alpha}$ is continuous on $\overline{\mathbf{D}}$ and vanishes only at 1 . Since $\left|\tilde{b}_{\alpha}(1)\right|=\tilde{a}_{\alpha}(-1)=1$, there exist $\delta>0$ such that $\left|\tilde{b}_{\alpha}(z)\right|>1 / 2$ on $D_{1}=\overline{\mathbf{D}} \cap\{z:|z-1|<\delta\}$ and $\left|\tilde{a}_{\alpha}(z)\right|>1 / 2$ on $D_{2}=\overline{\mathbf{D}} \cap\{z:|z+1|<\delta\}$. Then the continuous function $\left|\tilde{b}_{\alpha}\right|^{2}+\left|\tilde{a}_{\alpha}\right|^{2}$ is positive on the compact set $\overline{\mathbf{D}} \backslash\left(D_{1} \cup D_{2}\right)$, so it is bounded from below by a strictly positive number $\varepsilon>0$. 
Remark 3.7. Since $\frac{1-z}{2}$ is the Pythagorean mate for $\frac{1+z}{2}$, we remark that it follows from [4] that for $\alpha>0$,

$$
\mathcal{H}\left(\left(\frac{1+z}{2}\right)^{\alpha}\right)=\mathcal{H}\left(\frac{1+z}{2}\right)=c+(1-z) H^{2}
$$

as sets.

Finally, we remark that if $u$ is a finite Blaschke product and $b_{\alpha}$ is given by (2.2), then

$$
\mathcal{H}\left(u b_{\alpha}\right)=\mathcal{H}\left(b_{\alpha}\right)
$$

Since every function in $\mathcal{H}(u)$ is holomorphic in $\overline{\mathbf{D}}$ (see, e.g. [6, Sec. 14.2]) and $\mathcal{H}\left(b_{\alpha}\right)$ is invariant under multiplication by functions holomorphic in $\overline{\mathbf{D}}$ (see, e.g. [12, (IV-6)]), (3.4) follows from the equality

$$
\mathcal{H}\left(u b_{\alpha}\right)=\mathcal{H}(u)+u \mathcal{H}\left(b_{\alpha}\right)
$$

\section{References}

[1] Chevrot, N., D. Guillot, and T. Ransford: De Branges-Rovnyak spaces and Dirichlet spaces. - J. Funct. Anal. 259:9, 2010, 2366-2383.

[2] Costara, C., and T. RAnsford: Which de Branges-Rovnyak spaces are Dirichlet spaces (and vice versa)? - J. Funct. Anal. 265:12, 2013, 3204-3218.

[3] Duren, P.: Theory of $H^{p}$ spaces. - Academic Press, New York-London, 1970.

[4] Fricain, E., A. Hartmann, and W. T. Ross: Concrete examples of $\mathcal{H}(b)$ spaces. - Comput. Methods Funct. Theory 16:2, 2016, 287-306.

[5] Fricain, E., A. Hartmann, and W. T. Ross: Range spaces of co-analytic Toeplitz operators. - Canad. J. Math. 70:6, 2018, 1261-1283.

[6] Frichin, E., and J. Mashreghi: The theory of $H(b)$ spaces. Vol. 1, 2. - Cambridge Univ. Press, Cambridge, 2016.

[7] Frichin, E., and J. Mashreghi: Boundary behavior of functions in the de Branges-Rovnyak spaces. - Complex Anal. Oper. Theory 2:1, 2008, 87-97.

[8] EAnucha, B., and M. NowAK: De Branges-Rovnyak spaces and generalized Dirichlet spaces. - Publ. Math. Debrecen 91, 2017, 171-184.

[9] Nikolski, N. K.: Operators, functions and systems: an easy reading. Volume 1: Hardy, Hankel and Toeplitz. - Math. Surveys Monogr. 92, Amer. Math. Soc., Providence, RI, 2002.

[10] Sarason, D.: Doubly shift-invariant spaces in $H^{2}$. - J. Operator Theory 16, 1986, 75-97.

[11] Sarason, D.: Kernels of Toeplitz operators. - In: Toeplitz operators and related topics (Santa Cruz, CA, 1992), Oper. Theory Adv. Appl. 71, Birkhäuser, Basel, 1994, 153-164.

[12] Sarason, D.: Sub-Hardy Hilbert spaces in the unit disc. - John Wiley and Sons Inc., New York, 1994.

[13] SARAson, D.: Local Dirichlet spaces as de Branges-Rovnyak spaces. - Proc. Amer. Math. Soc. $125: 7,1997,2133-2139$.

[14] Sarason, D.: Unbounded Toeplitz operators. - Integral Equations Operator Theory 61, 2008, 281-298.

Received 24 May 2018 • Accepted 23 October 2018 